Onkologie 1981;4:3

\title{
Impressum, Vol. 4, No. 1, 1981
}

\section{ONKOLOGIE}

Zeitschrift für Krebsforschung u. -behandlung

unter Fortführung der »österreichíschen Zeitschrift für Onkologie«

Band 4, Nummer 1, Februar 1981

Impressum

S. Karger

Verlag für Medizin und Naturwissenschaften GmbH,

Postfach 2, D-8034 Germering

Wissenschaftlicher Beirat

H. W. Bauer, München; H. Denck, Wien; V. Diehl, Hannover; F. Douwes, Göttingen; P. Drings, Heidelberg; S. Eckhardt, Budapest; H. Ehrhart, München; J. Fischer, Mainz; W. M. Gallmeier, Nürnberg; H. Gerhartz, Berlin; E. H. Graul, Marburg/Lahn; R. Gross, Köln; H. Heimpel, Ulm; K. P. Hellriegel, Köln; Ch. Her-farth, Ulm; J. H. Holzner, Wien; R. Hünig, Basel; W. Hunstein, Heidelberg; H. O. Klein, Köln; H. Löffler, Lahn Gießen; U. Mohr, Hannover; F.-L. Müller, Bonn; K. Munk, Heidelberg; G. P. Murphy, Buffalo (N.Y.); G. Nagel, Göttingen; A. Pfleiderer, Freiburg; Mildred Scheel, Bonn; E. Scherer, Essen; S. Seeber, Essen; H. J. Senn, St. Gallen; A. Stacher, Wien; St. Tanneberger, Berlin/ DDR; W. Vahlensieck, Bonn; H. D. Waller, Tubingen; M. Wan-nenmacher, Freiburg; W. Willmanns, München; K. Wilms, Tubingen; K. zum Winkel, Heidelberg; H. Wrba, Wien.

Verantwortliche Schriftleitung z. Zt. S. Eckhardt, Budapest J. H. Holzner, Wien G. A. Nagel, Göttingen

Die Zeitschrift erscheint zweimonatlich; pro Jahr erscheint 1 Band zu je 6 Heften.

Bezugspreis für Jahrgang 4, 1981 DM 112,-/ÖS 784,-/SFr. 112,-. 1 Einzelheft kostet DM 23,/ÖS 161,-/SFr. 23,-, einschließlich MwSt., zuzüglich Postgebühren.

Der Abonnementspreis ist im voraus zahlbar. Die Lieferung der Zeitschrift läuft weiter, wenn sie nicht spätestens 4 Wochen vor Abschluß eines Bandes abbestellt wird.

Abonnementsbestellungen können bei jeder Buchhandlung, bei der Post oder direkt beim Verlag aufgegeben werden: Bundesrepublik Deutschland/österreich: S. Karger GmbH, Postfach 2, D8034 Germering/München, Tel: (089) 844021, Telex: 524865 D, Postscheckkonto: München 400 80-807 Schweiz: S. Karger AG, Allschwilerstr. 10, Postfach, CH-4009 Basel, Tel.: (061) 390880, Telex62652 CH

\section{Anzeigen}

S. Karger Verlag für Medizin und Naturwissenschaften GmbH, Postfach 2, 8034 Germering, Telefon (089) 844021.

Für den Inhalt außerhalb des redaktionellen Tales (insbesondere Anzeigen, Industrieinformationen, Pressezitate und Kongreßinfor-mationen) übernehmen Herausgeber und Verlag keine Gewähr. 
Alle Rechte, insbesondere das Recht der Vervielfältigung und Mikrokopie sowie der Übersetzung, vorbehalten. Nachdruck, auch auszugsweise, nur mit Genehmigung des Verlages.

(C) Copyright 1981 by S. Karger Verlag für Medizin und Naturwissenschaften GmbH, Postfach 2, D-8034 Germering

Satz und Druck, Walter Biering GmbH, Grafischer Betrieb, 8000 München 45.

Offiziclles Organ

der Deutschen Gesellschaft für Hämatologie und Onkologie

und der

österreichischen Gesellschaft für Hämatologie und Onkologie

Off izielles Organ

der österreichischen Krebsgesellschaft- Krebsliga 